\title{
The diagnosis of traumatic brain injury on the battlefield
}

\section{Kara E. Schmid ${ }^{* \dagger}$ and Frank C. Tortella ${ }^{\dagger}$}

Brain Trauma Neuroprotection and Neurorestoration Department, Center for Military Psychiatry and Neuroscience, Walter Reed Army Institute of Research, Silver Spring, MD, USA

\section{Edited by:}

Stefania Mondello, University of

Florida, USA

Reviewed by:

Mattias Sköld, Uppsala University, Sweden

Amade Bregy, University of Miami, USA

\section{${ }^{*}$ Correspondence:}

Kara E. Schmid, Center for Military Psychiatry and Neuroscience, Walter Reed Army Institute of Research, 503 Robert Grant Avenue, Silver Spring, MD 20910-7500, USA.

e-mail: kara.schmid@us.army.mil

tThe views of the authors do not purport or reflect the position of the Department of the Army or the Department of Defense (para 4-3, AR 360-5).
The conflicts in Iraq and Afghanistan have placed an increased awareness on traumatic brain injury (TBI). Various publications have estimated the incidence of TBI for our deployed servicemen, however all have been based on extrapolations of data sets or subjective evaluations due to our current method of diagnosing a TBI. Therefore it has been difficult to get an accurate rate and severity of deployment related TBIs, or the incidence of multiple TBIs our service members are experiencing. As such, there is a critical need to develop a rapid objective method to diagnose TBI on the battlefield. Because of the austere environment of the combat theater the ideal diagnostic platform faces numerous logistical constraints not encountered in civilian trauma centers. Consequently, a simple blood test to diagnosis TBI represents a viable option for the military. This perspective will provide information on some of the current options for TBI biomarkers, detail concerning battlefield constraints, and a possible acquisition strategy for the military. The end result is a non-invasive TBI diagnostic platform capable of providing much needed advances in objective triage capabilities and improved clinical management of in-Theater TBI.

Keywords:TBI, military, biomarkers, diagnosis, concussion

\section{THE TBI PROBLEM}

In the early years of the wars in Afghanistan (Operation Enduring Freedom, OEF) and Iraq (Operation Iraqi Freedom, OIF), there appeared to be an increase in the numbers of causalities sustaining a traumatic brain injury (TBI). By early 2005 TBI was being called the "signature wound of war in Iraq" as cases of soldiers suffering from TBI were appearing in National newspapers, such as USA Today (Okie, 2005; Zoroya, 2005). The increased awareness and emphasis of TBI in the military population spurned a movement to identify and collect data on the incidence of TBI in our deployed forces.

The incidence of TBI in the deployed forces varies depending upon the type of information collected. One initial study examined a cohort of casualties that were wounded in OEF/OIF from 2001 to 2005 . The study reported that approximately $30 \%$ of wounds were to the head and neck area with $8 \%$ of the total attributed as head wounds (Owens et al., 2008). However, this study was based on casualties treated for wounds, and excluded those that were returned to duty within $72 \mathrm{~h}$, thus potentially missing TBI cases that were mild and did not accompany an open wound. The RAND Corporation's "Invisible Wounds of War" collected data from April 2007 to January 2008, part of which included a telephone survey of 1,965 previously deployed persons. From this survey they reported that $19.5 \%$ of the previously deployed persons suffered from a "probable TBI." They further estimated that of the 1.64 million deployed service members (at that time), $19.5 \%$ of them, or 320,000 have suffered a TBI (Tanielian and Jaycox, 2008). However, data collected from the Armed Forces Health Surveillance Center show that by the end of 2008 there had only been approximately 130,000 clinically confirmed cases of TBI. In addition, the number of TBI diagnosis rose sharply between the years of 2005 thru 2009, specifically in the cases of mild TBI (mTBI; http://www.health.mil/Research/TBI_Numbers.aspx). Is this rise in TBI a real rise, or an increase in TBI awareness and improved vigilance for detecting TBI? Clearly there appears to be a real challenge on getting an accurate estimate of the actual incidence of TBI experienced by our deployed Forces.

\section{DIAGNOSING TBI}

One of the issues creating this challenge lies in the current limitations in the diagnosis of TBI, specifically for the military. In general, the diagnosis of TBI relies on a clinician to accurately interpret a patient's signs and symptoms of injury, often through some type of self-report from the patient and possibly corroborated by a witness. In the case of a penetrating brain injury, the signs and symptoms are more straightforward. However, with closed-head injuries, especially mTBIs/concussions, the symptoms are often not as straightforward or clear. Typically the initial evaluation of a possible TBI captures the patients Glasgow Coma Scale (GCS), the length of loss of consciousness, alteration of consciousness/mental state, and/or post-traumatic amnesia (Harrington et al., 1993; Meyer et al., 2010). If certain symptoms are present the clinician can further evaluate the injury by image analysis of the brain via a computed tomography (CT) scan or even magnetic resonance imaging (MRI), both of which have their own limitations in the ability to detect TBI, especially mTBI (Chastain et al., 2009; Mondello et al., 2011a; Prabhu, 2011). Besides the image analysis, most of the tools to aid in the diagnosis of TBI are subjective and as stated above involve self-reporting. Hence, there are no true objective measures available to determine that the brain indeed has been injured, thus the ability to diagnose closed-head TBI, especially those of the mild-moderate severity, is at best limited by 
available methods. However, the ability to diagnosis a TBI can also be limited by the setting.

The civilian environment and the military (deployed) environment are vastly different. In a civilian setting, if someone experiences a head injury from a motor vehicle accident, fall, or a sporting event, the event is often witnessed; they are quickly transported to the local medical facility and promptly evaluated by a medical professional, sometimes with access to the latest imaging technology. In the military setting, there is often some type of battle taking place either before, during, or after, when someone experiences a head injury. By its very nature the event is dangerous, and often chaotic and loud, occurring in remote locations and possibly lasting hours before casualties can be evaluated by a clinician. In addition, the military casualty can and often will experience multiple serious injuries (Kelly et al., 2008; Owens et al., 2008) and the evaluation of a potential head injury can take a back seat to life saving interventions, possibly missing the treatment window for the TBI. Further, evacuation priorities and logistics may impact the ability to evaluate all suspected mTBI cases to the same extent as a civilian setting as the ability to image the brain is only located at specific locations in the theater of operations. Clearly the military setting creates another layer of difficulty and complexity in the ability to diagnose TBI.

\section{ON THE BATTLEFIELD}

Due to the complexities of war and of TBI, it has been extremely difficult to get a handle on the rate of TBIs experienced during a deployment, not to mention the severity of TBIs or the incidence of multiple TBIs our service members are actually experiencing, or not experiencing. One of the major reasons is the lack of a method to objectively determine if the brain has been injured. In 2006 the Military Acute Concussion Evaluation (MACE) was add to the list of tools to screen casualties (Meyer et al., 2010). While the MACE has been well integrated into the military medical evaluation, it still relies on subjective recall of the events, may be affected by fatigue as other neuropsychological tests and has shown low sensitivity when administered greater than $12 \mathrm{~h}$ (Coldren et al., 2010). Thus there remains a gap for the ability to objectively measure brain injury with a method that is not impacted by other factors such as extra-cranial injury (i.e., polytrauma), stress, fatigue, or battlefield conditions.

Due to the logistic constraints faced on a battle field, any method involving a piece of equipment, must meet additional requirements sometimes not faced in civilian medical care. The battlefield is often a very austere environment. The most restrictive environments are often isolated locations where power supply is lacking or being provided by a generator which must sustain all of the electrical needs for the deployed force. Since units often need to remain quickly mobile, the footprint of the unit is limited, so space, and weight of equipment is a priority concern. In addition, if medical refrigeration exists, it is typically small and in high demand and there is little hope of having specialized reagents such as deionized water. Further consideration is given to the shelf-life of a piece of equipment and its ability to sustain high altitude, large temperature, and/or humidity fluctuations and a considerably dusty and dirty environment. Taking all of these issues into consideration, the ideal method for the diagnosis of TBI would be quick, simple, easy to obtain, not rely on self-report of symptoms, and be portable. In addition, the results of the test should be able to differentiate the severity of injury and in a most ideal world, be predictive of some level of clinical outcome.

\section{OBJECTIVE DIAGNOSTIC TEST FOR TB|}

In recent years there have been a number of technologies under development to objectively aid in the diagnosis of TBI (Marion et al., 2011). Some of these include advances in MRI (Kumar et al., 2010; Prabhu, 2011), quantitative electroencephalogram (EEG; Nuwer et al., 2005), visual tracking (Maruta et al., 2010), and serum based biomarkers of brain injury (Dash et al., 2010). Using the logistical constraints mentioned above and the need for the test to not be confounded by conditions of deployment (sleep deprivation, stress, fatigue, etc.), one of the more promising options is the development of a simple blood test to detect brain specific proteins after a TBI. Blood based tests have been successful in the diagnosis of other disease conditions such as cardiac disease and cancer, so it is possible biomarkers could be identified for TBI as well.

The TBI community has been actively engaged in the discovery of biomarkers for TBI in the last decade. A number of review articles have captured the pros and cons of various potential markers (Dash et al., 2010; Mondello et al., 2011a). Some of the more promising candidates have been tested in human clinical trials of TBI patients, most of them in severe TBI (Hergenroeder et al., 2010; Liliang et al., 2010; Mondello et al., 2010, 2011b, 2012a,b; Vos et al., 2010; Brophy et al., 2011; Stein et al., 2011, 2012; Gong et al., 2012). A number of proteins of interest are markers that are not solely found in the brain (i.e., cytokines, growth factors, interleukins; Hergenroeder et al., 2010; Stein et al., 2011; Gong et al., 2012). While these may provide clinical utility in situations of head trauma with no confounding injuries, this is typically not the case in the military combat causality. However, a number of markers of interest are more brain specific, to include S100B, glial fibrillary acidic protein (GFAP), Ubiquitin C-terminal hydrolase L1 (UCHL1), Neuron Specific Enolase (NSE), spectrin breakdown products (SBDP), and Tau (Siman et al., 2009; Liliang et al., 2010; Mondello et al., 2010, 2011b, 2012a,b; Vos et al., 2010; Brophy et al., 2011; Stein et al., 2012).

Most of the clinical trials have been conducted with severe TBI patients, but a few have also included moderate and mTBI patients as well and show promise (Honda et al., 2010; Papa et al., 2012a,b; Topolovec-Vranic et al., 2011; Egea-Guerrero et al., 2012). In an early study of S100B in mTBI subjects, S100B serum levels were significantly different between mTBI patients and uninjured controls (Nygren De Boussard et al., 2004). However, S100B is also increased in other extra-cranial injuries, making less than ideal in the military environment (Savola et al., 2004). Promising recent studies include a study on moderate-mTBI $(\mathrm{GCS} \leq 12)$, that demonstrated that the levels of GFAP in patient serum were able to significantly differentiate not only between TBI patients and uninjured controls, but also between TBI patients and trauma controls (trauma injury without head injury). Further, when TBI groups were dichotomized into traditional groups of mTBI (GCS 13-15) and moderate TBI (GCS 9-12), the level of serum GFAP were significantly different between groups (Papa et al., 2012a). Similar results were demonstrated with serum levels of UCH-L1 differentiating between mTBI, moderate TBI, normal, and trauma controls (Papa et al., 2012b). Another study recently characterized 
the differences in areas under the curve (AUC) for sensitivity and specificity of GFAP, S100B, and NSE in TBI patients (GCS range 5-14) determined by positive CT findings. GFAP performed the best with sensitivity set to $100 \%$ and the corresponding specificity was $88.9 \%$ on day one compared to $\mathrm{S} 100 \mathrm{~B}$ with a specificity of $27.8 \%$ and NSE with a specificity of $22.2 \%$ on day one (Honda et al., 2010).

\section{TBI BIOMARKER PLATFORMS FOR THE FIELD}

Although recent trials have indicated that it is possible to detect TBI biomarkers in the serum of injured patients, all of the studies have taken place in civilian centers and were able to use a research laboratory platform for analysis. Typically this platform is a standard enzyme-linked immunosorbent assay (ELISA) which has a number of limitations for use in a combat environment. First, the typical assay requires multiple pieces of equipment (reader, incubator, and automated washing machine), refrigeration of reagents, and deionized water. Second, the assay can be lengthy to run, taking as little as $4 \mathrm{~h}$ and as many as $24 \mathrm{~h}$. Third, the standard assay plate consists of 96 wells, which could equate to wasting two-thirds of a plate if there is only one sample to assay. However, when we consider the needs for our military applications the assay in an ELISA platform is indeed the most mature in development and testing of clinical samples. Similarly this platform could potentially have greater sensitivity (lower limits of detection) and serve as a benchtop in a reference laboratory. In addition, porting the standard ELISA assay onto an automated benchtop platform could cut down on some of the size and weight of the system.

On the other end of the spectrum would be the development of a hand-held device that performs similar to a TBI "pregnancy test." Such hand-held systems would be lightweight, portable, and require little logistical support. The assay time and waste would likely be reduced as well. However, these devices do not exist today for the TBI biomarkers that have been examined in clinical studies; therefore they are the least mature in development and Food and Drug Administration (FDA) approval timeline. In addition, it is possible that this platform could suffer in the limit of detection.

As mentioned above, the development of a device platform for the military environment presents unique challenges with different restrictions upon instrumentation at each level of care. The initial level of combat casualty care is located close to the point of injury such as a Battalion Aid Station (BAS). This level of care is the most remote and has the highest logistical constraints concerning power, refrigeration, and footprint. In addition, this level of care does not have much capacity, if any, to hold patients for treatment and evaluation. The most robust level of care on the battlefield is located at a combat support hospital (CSH) or a field hospital. It includes specialist diagnostic resources, specialist surgical and medical capabilities, and operational stress management teams. The holding capacity is sufficient to allow diagnosis, treatment, and holding of those patients who can receive total treatment and be returned to duty. However, it is still a deployed environment faced with the same challenges of reduced and restricted footprint, especially for power and refrigeration. For the highest level of definitive care, patients are evacuated back to fixed facility hospitals such as Landstuhl Regional Medical Center or Walter Reed
National Military Medical Center. These hospitals operate as any civilian medical facility with few, if any, logistical constraints.

\section{MILITARY ACQUISITION APPROACH}

Given (1) the importance of providing an objective test to aid in the diagnose of TBI, (2) the level of maturity of platforms to evaluate TBI biomarkers, and (3) the unique abilities and constraints on each level of possible medical care, the best approach to solve this problem may be an phased acquisition approach (Mondello et al., 2011a; Figure 1). The first phase of development/fielding should focus on the technology that is the most mature, and potentially fieldable, not only in terms of platform logistics but also in clinical testing and familiarity with the FDA. Therefore, Phase I could focus on an automated benchtop system suitable for use in a fixed medical facility. Although this system is the most complex and logistically intensive, it is the most similar to the standard research ELISA that have been employed in previous testing of clinical samples. In addition, it could possibly have the highest level of sensitivity for detection of biomarkers and ultimately serve as a reference platform for confirmatory testing (higher diagnostic specificity). Phase II could be a small single assay point-of-care system, such as a physician's office or a field hospital. Phase II systems would ideally have the ability to screen for the presence of mild-moderate TBI and differentiate the level of severity for all TBIs. Ideally Phase II systems would be more portable than Phase I systems, have the availability of battery operation and require less refrigeration of reagents. Phase III systems could be a miniaturized hand-held system, most suitable for use by emergency medical personnel or personnel deployed in the remote and more austere locations on the battlefield, such as a BAS. These systems would ideally use whole blood from a finger stick to screen for the presence of TBI. They would use little power supply and require no refrigeration. Currently, Phase III systems are the least mature in development and testing for TBI biomarkers, but would provide the most use for screening of casualties (highest diagnostic sensitivity).

If all three Phases of systems were developed and deployed, one could image a scenario that starts with screening casualties for the presence of TBI biomarkers immediately after an event. Screening tests can typically have higher diagnostic sensitivity than diagnostic specificity (Ruan et al., 2009; i.e., more false positives than false negatives). Using a screening test designed in this manner will ensure you do not miss a casualty with a possible TBI, however you will have more people identified as having a TBI that could later be ruled out. The casualty could be evacuated back to the next higher level of care that can provide a more robust confirmatory test for the TBI biomarkers. At the highest level of care, the test could be more confirmatory and have a higher diagnostic specificity than sensitivity (lower number of false positive) and essentially rule in those with TBI and determine which casualties were false positives for the screening test. In addition, TBI biomarker assays at the highest level of care could also be used to possibly determine what type of injury has occurred and monitor if the injury is getting worse over time or better with treatment (Mondello et al., 2011a). This scenario also fits well with the current military levels of medical care on the battlefield. The biomarker assay screen (Phase I/II) could be completed during the initial assessment after 


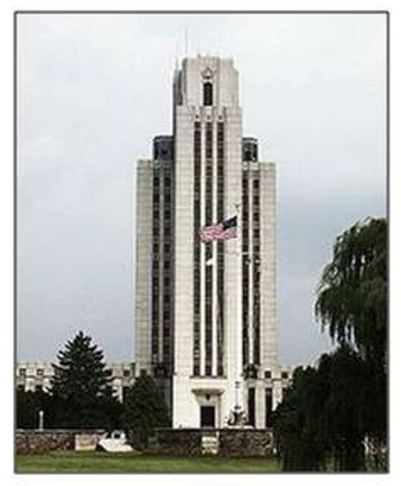

\section{Potential Placement of TBI Diagnostic Assay Systems}

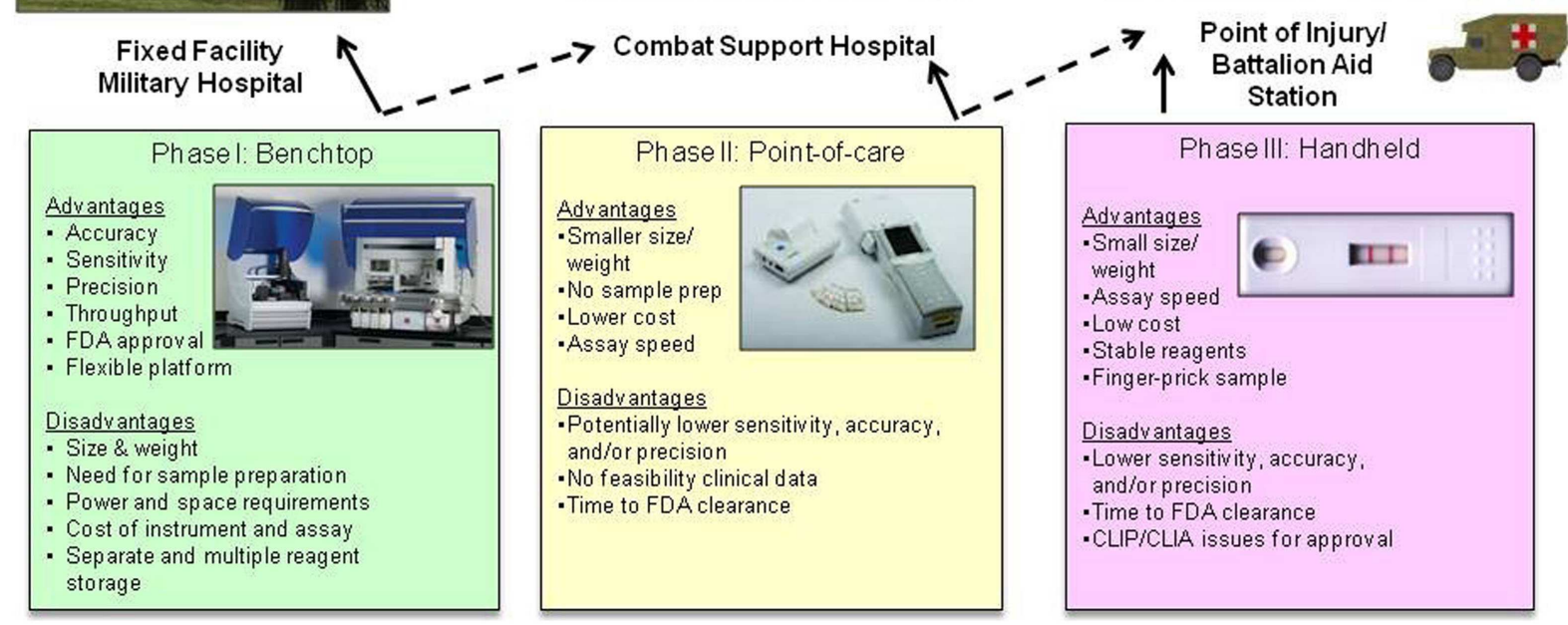

FIGURE 1 | Potential placement of TBI diagnostic assay systems. Each potential device has advantages and disadvantages that constrain its use in the military medical system. Solid lines represent ideal placement of each system, dashed lines represent possible placement of each system depending on logistical support. All device pictures are representative examples of commercially available devices, but do not indicate the use of TBI biomarkers on each device. Pictured benchtop device is Dynex Technologies DS2 ${ }^{\mathrm{TM}}$ and DSXTM(http://www.dynextechnologies.com), pictured point-of-care device is Abbott Laboratories ISTAT ${ }^{\circledR}$ (http://www.abbottpointofcare.com)

injury, where the other screening test, the MACE is performed. The biomarker assay confirmatory test (Phase III) could be performed at a field hospital where other more extensive diagnostic tools are available, such as a CT.

\section{SUMMARY}

Overall the current methods to diagnosis a TBI could be improved with the development and addition of a non-invasive, objective test for the presence of TBI. Current research and development in the field of biomarkers give hope the development of such an objective diagnostic test. However, military conditions

\section{REFERENCES}

Brophy, G. M., Mondello, S., Papa, L., Robicsek, S. A., Gabrielli, A., Tepas, J. III, Buki, A., Robertson, C., Tortella, F. C., Hayes, R. L., and Wang, K. K. (2011). Biokinetic analysis of ubiquitin C-terminal hydrolase-L1 (UCH-L1) in severe traumatic brain injury patient biofluids. J. Neurotrauma 28, 861-870.

Chastain, C. A., Oyoyo, U. E., Zipperman, M., Joo, E., Ashwal, S., Shutter,
L. A., and Tong, K. A. (2009). Predicting outcomes of traumatic brain injury by imaging modality and injury distribution. J. Neurotrauma 26, 1183-1196.

Coldren, R. L., Kelly, M. P., Parish, R. V., Dretsch, M., and Russell, M. L. (2010). Evaluation of the military acute concussion evaluation for use in combat operations more than 12 hours after injury. Mil. Med. 175, $477-481$.

contain logistical constraints which may require different platforms for different levels of care. Development of this non-invasive TBI diagnostic platform applicable to all levels of military care would provide much needed advances in objective triage capabilities and improved clinical management of in-Theater TBI. The ability to objectively determine the occurrence of an initial $\mathrm{mTBI} /$ concussion and the incidence of multiple mTBIs on our front lines of defense is critical to the success of our military operations, and to the long term health of our warfighters. Likewise, improved TBI diagnosis will also significantly advance the management of civilian patients.

Dash, P. K., Zhao, J., Hergenroeder G., and Moore, A. N. (2010). Biomarkers for the diagnosis, prognosis, and evaluation of treatment efficacy for traumatic brain injury. Neurotherapeutics 7 , $100-114$.

Egea-Guerrero, J. J., RevueltoRey, J., Murillo-Cabezas, F. Munoz-Sanchez, M. A., VilchesArenas, A., Sanchez-Linares, P., Dominguez-Roldan, J. M., and
Leon-Carrion, J. (2012). Accuracy of the S100beta protein as a marker of brain damage in traumatic brain injury. Brain Inj. 26, 76-82.

Gong, D., Hao, M., Liu, L., Liu, C., Dong, J., Cui, Z., Sun, L., Su, S., and Zhang, J. (2012). Prognostic relevance of circulating endothelial progenitor cells for severe traumatic brain injury. Brain Inj. 26, 291-297. 
Harrington, D. E., Malec, J., Cicerone, K., and Katz, H. T. (1993). Current perceptions of rehabilitation professionals towards mild traumatic brain injury. Arch. Phys. Med. Rehabil. 74, 579-586.

Hergenroeder, G. W., Moore, A. N., Mccoy, J. P. Jr., Samsel, L., Ward, N. H. III, Clifton, G. L., and Dash, P. K. (2010). Serum IL-6: a candidate biomarker for intracranial pressure elevation following isolated traumatic brain injury. J. Neuroinflammation 7 , 19.

Honda, M., Tsuruta, R., Kaneko, T., Kasaoka, S., Yagi, T., Todani, M., Fujita, M., Izumi, T., and Maekawa, T. (2010). Serum glial fibrillary acidic protein is a highly specific biomarker for traumatic brain injury in humans compared with S-100B and neuron-specific enolase. J. Trauma 69, 104-109.

Kelly, J. F., Ritenour, A. E., Mclaughlin, D. F., Bagg, K. A., Apodaca, A. N., Mallak, C. T., Pearse, L., Lawnick, M. M., Champion, H. R., and Holcomb, J. B. (2008). Injury severity and causes of death from operation Iraqi freedom and operation enduring freedom: 2003-2004 versus 2006. J. Trauma 64, S21-S26.

Kumar, R., Saksena, S., Husain, M., Srivastava, A., Rathore, R. K., Agarwal, S., and Gupta, R. K. (2010). Serial changes in diffusion tensor imaging metrics of corpus callosum in moderate traumatic brain injury patients and their correlation with neuropsychometric tests: a 2-year follow-up study. J. Head Trauma Rehabil. 25, 31-42.

Liliang, P. C., Liang, C. L., Weng, H. C., Lu, K., Wang, K. W., Chen, H. J., and Chuang, J. H. (2010). Tau proteins in serum predict outcome after severe traumatic brain injury. J. Surg. Res. 160, 302-307.

Marion, D. W., Curley, K. C., Schwab, K., Hicks, R. R., and mTBI Diagnostics Workgroup. (2011). Proceedings of the military mTBI Diagnostics Workshop, St. Pete Beach, August 2010. J. Neurotrauma 28, 517-526.

Maruta, J., Suh, M., Niogi, S. N., Mukherjee, P., and Ghajar, J. (2010). Visual tracking synchronization as a metric for concussion screening. $J$. Head Trauma Rehabil. 25, 293-305.

Meyer, K. S., Marion, D. W., Coronel, H., and Jaffee, M. S. (2010). Combat-related traumatic brain injury and its implications to military healthcare. Psychiatr. Clin. North Am. 33, 783-796.

Mondello, S., Muller, U., Jeromin, A., Streeter, J., Hayes, R. L., and Wang, K. K. (2011a). Blood-based diagnostics of traumatic brain injuries. Expert Rev. Mol. Diagn. 11, 65-78.

Mondello, S., Papa, L., Buki, A., Bullock, M. R., Czeiter, E., Tortella, F. C., Wang, K. K., and Hayes, R. L. (2011b). Neuronal and glial markers are differently associated with computed tomography findings and outcome in patients with severe traumatic brain injury: a case control study. Crit. Care 15, R156.

Mondello, S., Jeromin, A., Buki, A., Bullock, R., Czeiter, E., Kovacs, N., Barzo, P., Schmid, K., Tortella, F. C., Wang, K. K., and Hayes, R. L. (2012a). Glial neuronal ratio (GNR): a novel index for differentiating injury type in patients with severe traumatic brain injury. J. Neurotrauma 29, 1096-1104.

Mondello, S., Linnet, A., Buki, A., Robicsek, S., Gabrielli, A., Tepas, J., Papa, L., Brophy, G. M., Tortella, F., Hayes, R. L., and Wang, K. K. (2012b). Clinical utility of serum levels of ubiquitin C-terminal hydrolase as a biomarker for severe traumatic brain injury. Neurosurgery 70 , 666-675.

Mondello, S., Robicsek, S. A., Gabrielli, A., Brophy, G. M., Papa, L., Tepas, J., Robertson, C., Buki, A., Scharf, D., Jixiang, M., Akinyi, L., Muller, U., Wang, K. K., and Hayes, R. L. (2010). AlphaII-spectrin breakdown products (SBDPs): diagnosis and outcome in severe traumatic brain injury patients. J. Neurotrauma 27, 1203-1213.

Nuwer, M. R., Hovda, D. A., Schrader, L. M., and Vespa, P. M. (2005). Routine and quantitative EEG in mild traumatic brain injury. Clin. Neurophysiol. 116, 2001-2025.

Nygren De Boussard, C., Fredman, P., Lundin, A., Andersson, K., Edman, G., and Borg, J. (2004). S100 in mild traumatic brain injury. Brain Inj. 18, 671-683.

Okie, S. (2005). Traumatic brain injury in the war zone. N. Engl. J. Med. 352, 2043-2047.

Owens, B. D., Kragh, J. F. Jr., Wenke, J. C., Macaitis, J., Wade, C. E., and Holcomb, J. B. (2008). Combat wounds in operation Iraqi Freedom and operation Enduring Freedom. $J$. Trauma 64, 295-299.

Papa, L., Lewis, L. M., Falk, J. L., Zhang, Z., Silvestri, S., Giordano, P., Brophy, G. M., Demery, J. A., Dixit, N. K., Ferguson, I., Liu, M. C., Mo, J., Akinyi, L., Schmid, K., Mondello, S., Robertson, C. S., Tortella, F. C., Hayes, R. L., and Wang, K. K. (2012a). Elevated levels of serum glial fibrillary acidic protein breakdown products in mild and moderate traumatic brain injury are associated with intracranial lesions and neurosurgical intervention. Ann. Emerg. Med. 59, 471-483.

Papa, L., Lewis, L. M., Silvestri, S., Falk, J. L., Giordano, P., Brophy, G. M., Demery, J. A., Liu, M. C., Mo, J., Akinyi, L., Mondello, S., Schmid, K., Robertson, C. S., Tortella, F. C., Hayes, R. L., and Wang, K. K. (2012b). Serum levels of ubiquitin C-terminal hydrolase distinguish mild traumatic brain injury from trauma controls and are elevated in mild and moderate traumatic brain injury patients with intracranial lesions and neurosurgical intervention. J. Trauma 72, 1335-1344.

Prabhu, S. P. (2011). The role of neuroimaging in sport-related concussion. Clin. Sports Med. 30, 103-114.

Ruan, S., Noyes, K., and Bazarian, J. J. (2009). The economic impact of S-100B as a prehead CT screening test on emergency department management of adult patients with mild traumatic brain injury. J. Neurotrauma 26 , 1655-1664.

Savola, O., Pyhtinen, J., Leino, T. K. Siitonen, S., Niemela, O., and Hillbom, M. (2004). Effects of head and extracranial injuries on serum protein S100B levels in trauma patients. J. Trauma 56, 1229-1234; discussion 1234.

Siman, R., Toraskar, N., Dang, A., Mcneil, E., Mcgarvey, M., Plaum, J., Maloney, E., and Grady, M. S. (2009). A panel of neuron-enriched proteins as markers for traumatic brain injury in humans. J. Neurotrauma 26, 1867-1877.

Stein, D. M., Lindell, A., Murdock, K. R., Kufera, J. A., Menaker, J., Keledjian, K., Bochicchio, G. V., Aarabi, B., and Scalea, T. M. (2011). Relationship of serum and cerebrospinal fluid biomarkers with intracranial hypertension and cerebral hypoperfusion after severe traumatic brain injury. $J$. Trauma 70, 1096-1103.

Stein, D. M., Lindell, A. L., Murdock, K. R., Kufera, J. A., Menaker, J., Bochicchio, G., Aarabi, B., and Scalea, T. M. (2012). Use of serum biomarkers to predict cerebral hypoxia following severe traumatic brain injury. J. Neurotrauma 29, 1140-1149.

Tanielian, T., and Jaycox, L. H. (ed.). (2008). Invisible Wounds of War: Psychological and Cognitive Injuries, Their Consequences, and Services to Assist Recovery. Santa Monica, CA: RAND Corporation.

Topolovec-Vranic, J., PollmannMudryj, M. A., Ouchterlony, D., Klein, D., Spence, J., Romaschin, A., Rhind, S., Tien, H. C., and Baker, A. J. (2011). The value of serum biomarkers in prediction models of outcome after mild traumatic brain injury. J. Trauma 71 , S478-S486.

Vos, P. E., Jacobs, B., Andriessen, T. M., Lamers, K. J., Borm, G. F., Beems, T., Edwards, M., Rosmalen, C. F., and Vissers, J. L. (2010). GFAP and S100B are biomarkers of traumatic brain injury: an observational cohort study. Neurology 75, 1786-1793.

Zoroya, G. (2005). Brain injuries a legacy of war. USA Today (accessed March 3, 2005).

Conflict of Interest Statement: The authors declare that the research was conducted in the absence of any commercial or financial relationships that could be construed as a potential conflict of interest.

Received: 30 March 2012; accepted: 18 May 2012; published online: 12 June 2012.

Citation: Schmid KE and Tortella FC (2012) The diagnosis of traumatic brain injury on the battlefield. Front. Neur. 3:90. doi: 10.3389/fneur.2012.00090

This article was submitted to Frontiers in Neurotrauma, a specialty of Frontiers in Neurology.

Copyright (c) 2012 Schmid and Tortella. This is an open-access article distributed under the terms of the Creative Commons Attribution Non Commercial License, which permits non-commercial use, distribution, and reproduction in other forums, provided the original authors and source are credited. 